\title{
Correction to: Measurable residual disease after the first consolidation predicts the outcomes of patients with acute promyelocytic leukemia treated with all-trans retinoic acid and chemotherapy
}

\author{
Hideho Henzan ${ }^{1} \cdot$ Ken Takase $^{2} \cdot$ Tomohiko Kamimura $^{3} \cdot$ Yasuo Mori $^{4} \cdot$ Goichi Yoshimoto $^{4} \cdot$ Hiromi Iwasaki $^{2}$. \\ Koji Nagafuji ${ }^{5} \cdot$ Ryosuke Ogawa $^{6} \cdot$ Tetsuya Eto $^{1} \cdot$ Naoyuki Uchida $^{7} \cdot$ Tomoaki Fujisaki $^{8} \cdot$ Koji Kato $^{4} \cdot$ Mariko Minami $^{2,4}$. \\ Yoshikane Kikushige $^{4} \cdot$ Koichi Akashi $^{4} \cdot$ Toshihiro Miyamoto $^{4}$ (ㅁ) . for the Fukuoka Blood \& Marrow Transplantation \\ Group (FBMTG)
}

Published online: 8 July 2020

(c) Japanese Society of Hematology 2020

\section{Correction to: International Journal of Hematology https://doi.org/10.1007/s12185-020-02911-z}

In the original publication of the article, the "CNS involvement" in the last row under the column " $N=50$ " has been published incorrectly. The correct Table 1 is given in this correction.

Publisher's Note Springer Nature remains neutral with regard to jurisdictional claims in published maps and institutional affiliations.

The original article can be found online at https://doi.org/10.1007/ s12185-020-02911-z.

Toshihiro Miyamoto

toshmiya@intmed1.med.kyushu-u.ac.jp

1 Department of Hematology, Hamanomachi Hospital, Fukuoka, Japan

2 Department of Hematology, National Kyushu Medical Center, Fukuoka, Japan

3 Department of Hematology, Harasanshin Hospital, Fukuoka, Japan

4 Department of Medicine and Biosystemic Science, Kyushu University Graduate School of Medical Science, 3-1-1 Maidashi, Higashi-ku, Fukuoka 812-8582, Japan

5 Department of Hematology, Kurume University Hospital, Kurume, Japan

6 Department of Hematology, Japan Community Health Care Organization Kyushu Hospital, Kita-Kyushu, Japan

7 Department of Hematology, Toranomon Hospital, Tokyo, Japan

8 Department of Hematology, Matsuyama Red Cross Hospital, Ehime, Japan 
Table 1 Background patient characteristics and summary of induction therapy

\begin{tabular}{|c|c|c|c|c|}
\hline & $\begin{array}{l}\text { Total } \\
N=50\end{array}$ & $\begin{array}{l}\text { Low risk } \\
(N=27)\end{array}$ & $\begin{array}{l}\text { High risk } \\
(N=23)\end{array}$ & $P$ \\
\hline Age (range), years & $45(18-65)$ & $44(18-65)$ & $45(19-63)$ & $0.83 *$ \\
\hline Male/female & $24 / 26$ & $13 / 14$ & $11 / 12$ & $1 * *$ \\
\hline WBC at diagnosis, $\times 10^{9} / \mathrm{L}$ & $2.1(0.2-97.8)$ & $0.9(0.2-2.3)$ & $16.8(3.6-97.8)$ & $<0.001 *$ \\
\hline Platelet at diagnosis, $\times 10^{9} / \mathrm{L}$ & $32(6-170)$ & $42(6-170)$ & $25(11-74)$ & $0.0185 *$ \\
\hline$P M L-R A R A, \times 10^{5} / \mu \mathrm{gRNA}$ & $2.2(0.21-42.0)$ & $2.1(0.21-28.0)$ & $2.3(0.21-42.0)$ & $0.922 *$ \\
\hline PT (range), sec, normal range $10-14$ & $15.7(8.0-89.9)$ & $13.6(11.2-89.9)$ & $17.8(8.0-71.8)$ & $<0.001 *$ \\
\hline APTT (range), sec, normal range 24-39 & $29.5(21-281)$ & $28.9(22.8-120)$ & $29.6(21-281)$ & $0.444^{*}$ \\
\hline \multicolumn{5}{|l|}{ DIC } \\
\hline Yes & 43 & 21 & 22 & $0.107 * *$ \\
\hline No & 7 & 6 & 1 & \\
\hline \multicolumn{5}{|l|}{ Additional cytogenetic abnormality } \\
\hline Yes & 13 & 8 & 5 & $0.721 * *$ \\
\hline No & 37 & 19 & 18 & \\
\hline \multicolumn{5}{|l|}{ CNS involvement } \\
\hline Yes & 1 & 0 & 1 & $0.46 * *$ \\
\hline No & 49 & 27 & 22 & \\
\hline
\end{tabular}

$W B C$ white blood cell, $P T$ prothrombin time, APTT activated partial thromboplastin time, DIC disseminated intravascular coagulation, $C N S$ central nervous system

*Mann-Whitney $U$ test

**Fisher's exact test 